\title{
The impact of successive COVID-19 lockdowns on people mobility, lockdown efficiency, and municipal solid waste
}

\author{
Mengfan Cai ${ }^{1} \cdot$ Christophe Guy ${ }^{2} \cdot$ Martin Héroux ${ }^{3} \cdot$ Eric Lichtfouse $^{4,5}$ (D) Chunjiang An $^{1}$ (D)
}

Received: 28 April 2021 / Accepted: 9 July 2021 / Published online: 31 July 2021

(c) The Author(s), under exclusive licence to Springer Nature Switzerland AG 2021

\begin{abstract}
The COVID-19 pandemic has induced many issues for all societal sectors, in particular in the production and disposal of municipal solid waste. This may be because successive easing and reimposing of lockdown measures have deeply changed people's movements, consumers' behaviors and waste management. Previous studies have focused on the short-term effects of lockdowns on waste changes, yet there is little knowledge on waste variations during successive lockdowns and unlocking of various lockdown intensities. Moreover, the efficiency of lockdown and its relation to people's mobility in different countries are still not clear. Here, we studied the variations of amount and composition of municipal solid waste before the pandemic in 2019 and during the pandemic in 2020-2021 in USA, Brazil, Canada, UK, France and Italy. We used a stringency index and a composite mobility index to assess the lockdown intensity and people's movements. Results show that the mobility index sharply decreased with lockdown intensity, and enforcing measures were more efficient in France and Italy. Compared to 2019, prolonged lockdowns caused larger decreases in the quantity of commercial and construction wastes versus household waste. The initial implementation of lockdown or unlocking measures promoted inhabitants' consumption, generally leading to the increased waste amount, by about $9 \%$ for Trento and $12 \%$ for Montreal at the beginning of lockdown, respectively. Moreover, larger variations in the waste amount in Trento, from -25.3 to $9.8 \%$, were in line with higher lockdown intensity compared to those in Montreal, from -9.5 to $12.7 \%$, affected by people's mobility, consumers' behaviors and waste management.
\end{abstract}

Keywords COVID-19 $\cdot$ Lockdown $\cdot$ Resurgence $\cdot$ Municipal solid waste $\cdot$ Waste amount and composition

\section{Introduction}

The coronavirus disease 2019 (COVID-19) reported 172 million confirmed cases and 3.7 million deaths by June 3, 2021, globally (Google News 2021). The most affected

Chunjiang An

chunjiang.an@concordia.ca

1 Department of Building, Civil and Environmental Engineering, Faculty of Engineering and Computer Science, Concordia University, Montreal, Canada

2 Department of Chemical and Materials Engineering, Concordia University, Montreal, Canada

3 Department of Environment, City of Montreal, Montreal, Canada

4 Aix-Marseille Univ, CNRS, IRD, INRAE, CEREGE, Aix en Provence, France

5 State Key Laboratory of Multiphase Flow in Power Engineering, Xi' an Jiaotong University, Xi' an, China countries included the United States (USA), Brazil, India, Mexico, the United Kingdom (UK), Italy, Russian, and France (Google News 2021; Huo et al. 2021). Protective measures such as mask wearing, traffic restriction, social distancing, and home isolation have been globally implemented to reduce the spread of coronavirus (Wang et al. 2021). Many regions have experienced lockdowns since the beginning of this pandemic in March-April 2020, followed by gradual unlocking since May 2020. After this first pandemic wave, increasing economic and social activities have induced a second pandemic wave, as expected, in Euro-American countries since July 2020, and, in turn, new restrictions and lockdowns were imposed since September 2020. Lockdown periods have generally had positive impacts on environmental quality, with a notable decrease in urban air pollution (Wang et al. 2020a; Zambrano-Monserrate et al. 2020), yet there are concerns about the effect of lockdown on waste generation and recycling (Kulkarni and Anantharama 2020). Municipal solid waste (MSW) is an unavoidable by-product 
of human activity, and its recycling and recovery can facilitate the progression of a circular economy (Das et al. 2021). However, the COVID-19 pandemic has brought MSW systems severe challenges, such as the quantity variation and composition change of MSW (Vaverková et al. 2020; Vu et al. 2021; Yousefi et al. 2021). For instance, a significant escalation of medical waste was observed in some Asian cities and countries, such as India, during the lockdown compared to levels before the pandemic (Somani et al. 2020; You et al. 2020; Zand and Heir 2020). Nonetheless, the lockdown caused only a small waste rise in Singapore and even substantial reductions of MSW in India and Shanghai (Fan et al. 2020; Somani et al. 2020). Therefore, the effect of lockdown on waste dynamics can vary among regions due to distinct geographical, economic, and sociological characteristics. In particular, MSW production may be influenced by lockdown intensity and people's movements. Most studies mentioned above investigated the short-term effect of single lockdown on waste variations. The efficiency of lockdown and its relationship to people's mobility in different countries are still not clear. Here, we studied the effect of lockdown measures on people mobility, and the influence of successive lockdowns on waste variations in Euro-American regions.

\section{Experimental}

We selected six countries in Euro-America, including USA, Brazil, Canada, UK, France and Italy, in consideration of their large number of confirmed cases and deaths. Information on the timing and stringency of lockdown measures across the world was collected from the Oxford Coronavirus Government Response Tracker (OxCGRT 2021) and the COVID-19 Community Mobility Reports (2021). We also collected documents that provided the MSW amount in 2020-2021 or the variations in MSW amount between 2019 and 2020-2021 from news and municipal information. The City of Montreal in Canada and the City of Trento in Italy were selected as representative cities for America and Europe, respectively, to analyze the influence of varying pandemic lockdowns on waste variations during different lockdown periods (Trento Municipality 2021; City of Montreal 2020).

\section{Lockdown intensity and people mobility}

During the pandemic, the government lockdown intensity and people's movements changed with varying lockdowns, resulting in variations in the amount, composition and management of MSW. Here, we adopted a stringency index $\left(S_{I}\right.$, dimensionless) and a composite mobility index ( $M^{j}$, dimensionless) to represent the government lockdown intensity and people's movements, respectively. The $S_{I}$ was comprehensively calculated by nine response indicators (OxCGRT, 2021):

$S_{I}=\frac{\sum_{j=1}^{9} I_{j}}{9}$

where $I_{j}$ is the sub-index score for any given indicator $(j)$, such as closures of school, workplace, and public transport; restrictions on public events, public gatherings, internal movements, international travel, and public information campaigns; and stay-at-home requirements. The detailed equation for the $S_{I}$ can be found at OxCGRT (2021). In this study, the lockdown intensity was evenly classified into five levels based on the $S_{I}$, that is, level 1,2,3, 4 and 5 for $S_{I}$ of $0-20,20-40,40-60,60-80$ and 80-100, respectively.

If lockdown measures vary at the subnational level, $S_{I}$ is shown as the response level of the strictest subregion. This may result in the overestimation of the lockdown intensity of some cities. Mobility trends can show how peoples' movements have changed throughout the pandemic; thus, they were adopted to describe the specific lockdown intensity at different spatial scales. According to the COVID-19 Community Mobility Reports (2021), the mobility change $\left(M_{i}^{j}\right.$, $\%)$ in visitor numbers $\left(N_{i}\right)$ to specific categories of location between a COVID-19 pandemic period $(j)$ versus the baseline period before the pandemic was measured using Eq. 2:

$M_{i}^{j}(\%)=\frac{N_{\text {COVID }_{i}^{j}}-N_{\text {baseline }_{i}^{j}}}{N_{\text {baseline }_{i}^{j}}} \times 100 \%$

where $i$ indicates one of six broad categories of location, including residential places, grocery and pharmacy stores, workplaces, parks, transit stations, and retail and recreation. In this study, principal component analysis (PCA) was conducted using SPSS 16.0 to generate a composite mobility index for the comparison of different regions (Wang et al. 2020b). All the statistical analyses and figures were conducted and prepared using Microsoft Excel 2020, SPSS 16.0, and Origin Pro 9.1.

PCA results are shown in Table 1 and Supplementary Material's Tables S.1-6. Table S.1 shows that there is a linear correlation between six variables because the correlation coefficient between each group of variables is generally greater than 0.3. The overall KMO test coefficient is 0.707 ; the KMO test coefficient of each variable is greater than 0.5; and Bartlett's test result is $p<0.001$ (Tables S.2-S.3). These results mean that the data structure is reasonable, and the data can be extracted by principal components. Table 1 and Table S.4 suggest that only the eigenvalue of the top one principal component in this study is greater than 1, explaining $69.318 \%$ of the total data variation. Tables S.5-S.6 indicate the high explanatory ability of this principal component to six variables. Thus, the extracted principal component, 
Table 1 Total variance explained

\begin{tabular}{|c|c|c|c|c|c|c|}
\hline \multirow[t]{2}{*}{ Component } & \multicolumn{3}{|c|}{ Initial eigenvalues } & \multicolumn{3}{|c|}{ Extraction sums of squared loadings } \\
\hline & Total & $\%$ of Variance & Cumulative \% & Total & $\%$ of Variance & Cumulative \% \\
\hline 1 & 4.159 & 69.318 & 69.318 & 4.159 & 69.318 & 69.318 \\
\hline 2 & 0.981 & 16.355 & 85.673 & & & \\
\hline 3 & 0.528 & 8.808 & 94.481 & & & \\
\hline 4 & 0.201 & 3.346 & 97.827 & & & \\
\hline 5 & 0.088 & 1.470 & 99.297 & & & \\
\hline 6 & 0.042 & 0.703 & 100.000 & & & \\
\hline
\end{tabular}

Extraction method: principal component analysis
Table 2 MSW amount (tons) in Montreal and Trento in 2019

\begin{tabular}{lrrrr}
\hline City & \multicolumn{4}{l}{ Waste amount (tons) } \\
\cline { 2 - 5 } & Organic & Recyclable & \multicolumn{1}{l}{ Others } & \multicolumn{1}{c}{ Total } \\
\hline Montreal & 372,168 & 266,855 & 281,865 & 920,888 \\
Trento & 17,695 & 19,546 & 17,480 & 54,721 \\
\hline
\end{tabular}

the composite mobility index ( $M^{j}$, dimensionless), could be directly used as a newly generated variable for further data analysis.

\section{Amount of municipal solid waste}

In this study, organic and recyclable wastes were the major compositions, accounting for around $32 \%$ and $36 \%$ of the total waste amount (tons) in Trento and $40 \%$ and $29 \%$ in Montreal in 2019, respectively (Table 2). The recyclable waste included paper and cardboard, glass, metals and plastics in Montreal. Besides these waste types, lightweight packaging was additionally classified as recyclable waste in Trento. For the two cities, the amount of organic waste was the sum of food and green wastes. The impacts of the COVID-19 lockdown in changing waste amount and composition were evaluated by waste variations. The variations $\left(V_{i}^{j}, \%\right)$ of any type of MSW ( $W_{i}$, tons) between a COVID-19 pandemic period $(j)$ in 2020-2021 versus the same period in 2019 were calculated using Eq. 3:

$V_{i}^{j}(\%)=\frac{W_{\mathrm{COVID}_{i}^{j}}-W_{2019_{i}^{j}}}{W_{2019_{i}^{j}}} \times 100 \%$

\section{Results and discussion}

\section{Effects of lockdown intensity on people mobility}

We studied the effect of successive lockdowns and unlocking on the amount of municipal waste during the pandemic in 2020-2021, compared to 2019, in six countries. The mobility index represented the mobility change in visitor numbers throughout the pandemic, and the rapid variations in mobility were generally seen on weekends or holidays. Figure 1 shows that these countries have generally experienced four lockdown periods in 2020: pre-lockdown in January-February, first lockdown in February-May, unlocking in May-October, and second lockdown in November-December. However, Italy, France and USA implemented unlocking measures in January 2021, while the other three countries imposed stricter restrictions. Italy and France implemented the lockdown measures about half a month earlier than Canada and Brazil (Fig. 1).

Results show that the lockdown intensity increased sharply at the onset of the first lockdown for all countries (Fig. 1). During the first lockdown, the intensity was slightly higher, at level 5, for France and Italy, whereas the intensity of other countries was at level 4 . Then, during the first unlocking, the intensity decreased more for France and Italy, to level 3, than for other countries, with few or no decrease at level 4 until 2021. The second lockdown induced again a sharp increase of lockdown intensity for France and Italy. Overall, France and Italy experienced stronger variations in lockdown intensity.

The people mobility displayed in general reversed trends versus lockdown intensity, with more amplitude for France and Italy (Fig. 1). Mobility was slightly lower in major cities such as Trento and Montreal, versus the whole countries, which possibly indicates stricter enforcement in cities versus rural areas. Pearson correlation of stringency index and people mobility shows stronger relationships $(r)$ for France $(-0.817)$ and Italy $(-0.803)$ than for UK $(-0.758)$, USA $(-0.698)$, Brazil $(-0.600)$ and Canada $(-0.597)$ (Table S.7). Therefore, the lockdown was more efficient in France and Italy, which both applied a 'rest' period during the first unlocking. In other words, a too long period of intense locking is less effective, as clearly observed for Brazil, and to a lesser extent in USA and Canada where people mobility increased steadily despite high lockdown intensity. A possible explanation is that during prolonged lockdown, people get stressed by immobility, adapt and find a way to move. Overall, higher variations in lockdown intensity in France 

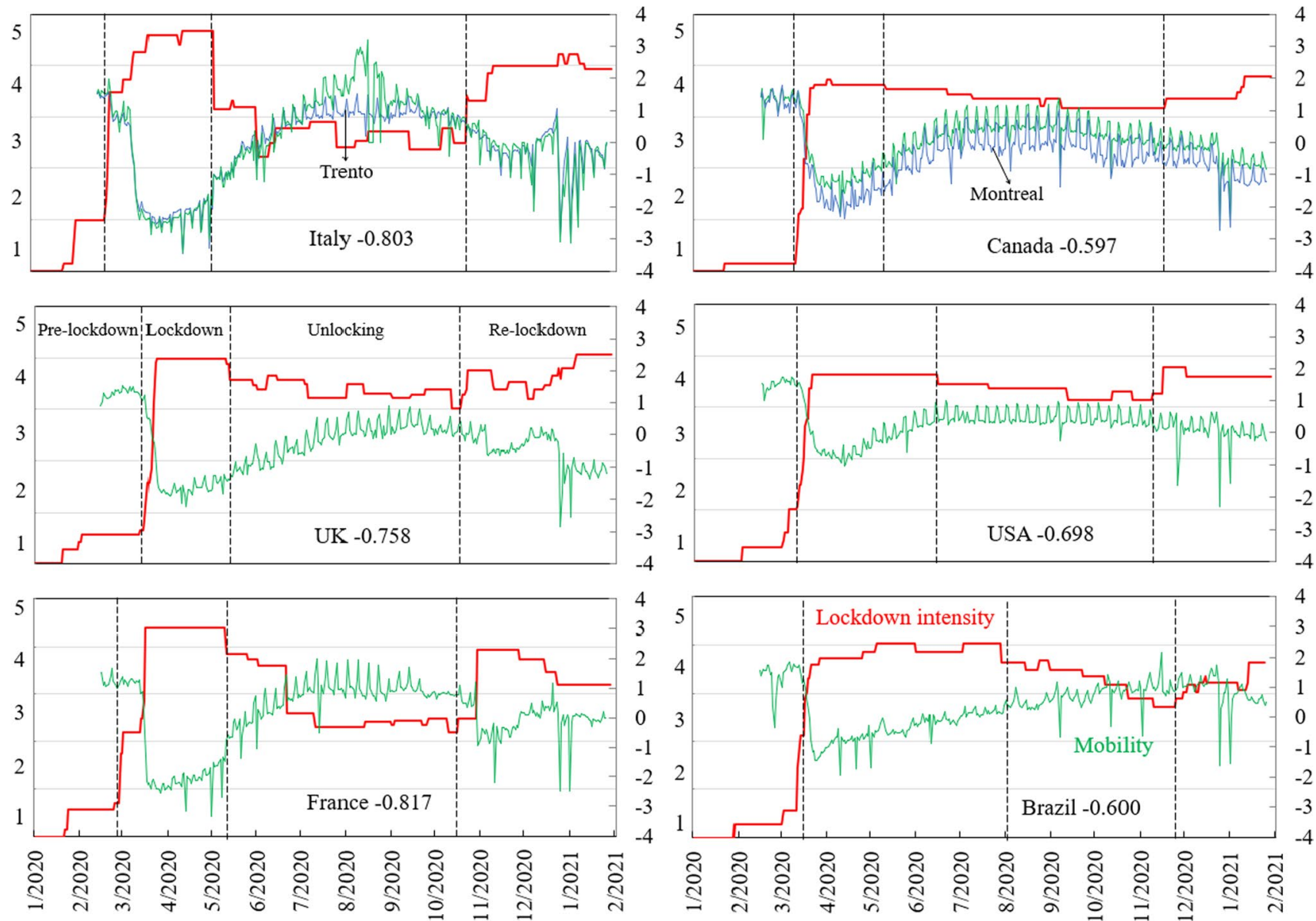

Fig. 1 Lockdown intensity and mobility index in 2020 in different cities/countries in Euro-America during the pandemic. The red lines show the lockdown intensities $(I)$ on the primary vertical axis which are evenly classified into five levels based on the stringency index, that is, level $1,2,3,4$ and 5 for $0-20,20-40,40-60,60-80$ and $80-100$, respectively. The green lines show the mobility indexes $\left(M^{j}\right)$ on the secondary vertical axis based on the national mobility changes

and Italy were more efficient at reducing the people mobility, versus prolonged lockdowns in UK, USA, Brazil and Canada.

\section{Waste amount dynamic during successive lockdowns}

Figure 2 shows the waste variations (\%) in the amount and composition of Montreal and Trento. Compared to the normal periods in 2019, significant decreases in total waste amount were observed in most of the months in 2020-2021 in Montreal (e.g., by 9.5\% in May 2020) and Trento (e.g., by $13.7 \%, 25.3 \%, 14.7 \%$ from March to May 2020 and $16.5 \%$ in January 2021). For the major waste composition, both organic and recyclable wastes showed similar trends as the total waste amount, down by $8-19 \%$. These results were observed when the national lockdown intensity is high, at in visitor numbers throughout the pandemic. Montreal in Canada and Trento in Italy were selected as the representative cities for America and Europe, respectively. Similarly, the blue lines show the $M^{j}$ values based on the city mobility changes in visitor numbers throughout the pandemic. The black vertical dashed lines show different lockdown periods in the pandemic according to $I$ and $M^{j}$ values

levels $4-5$, and the municipal mobility index is low, ranging from -2 to -1 (Fig. 1). Moreover, waste decreases in Trento were generally larger than those in Montreal because of the higher lockdown intensity and lower mobility index in Trento. This indicates that the significant reduction of people's movements could cause large decreases in waste amount. Notably, New York City and Stanford University communities in USA experienced significant decreases in commercial and construction wastes, nearly $50 \%$ and $60 \%$, respectively, in March (The Conversation, 2020; WasteAdvantage, 2020). Brno's household waste also decreased by $2.6 \%$ and $6.9 \%$ in April and May, respectively, and its commercial and industrial wastes dropped nearly $40 \%$ in June (Fan et al. 2020). UK's residual waste decreased by about $11 \%$, with commercial and industrial waste down by about 40\% during April-June (Tolvik 2020). These results indicate that prolonged lockdowns caused larger decreases in 


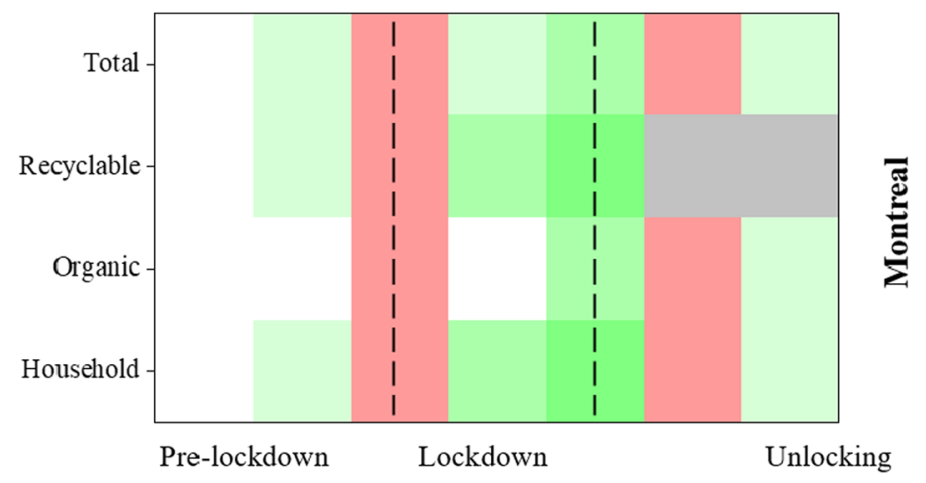

Re-lockdown

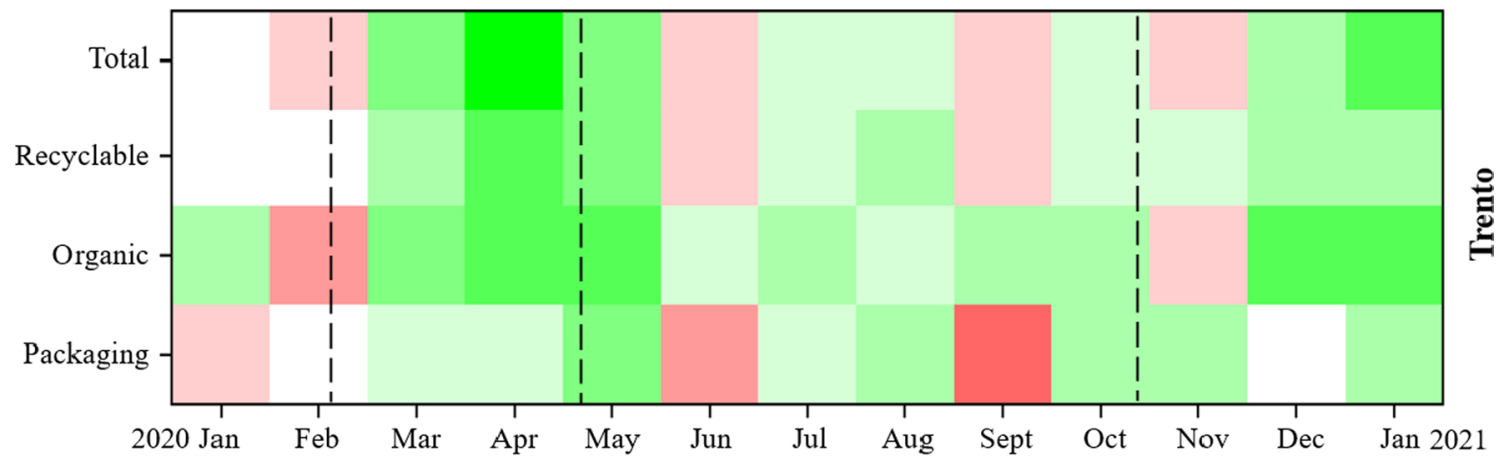

Waste variations (\%)

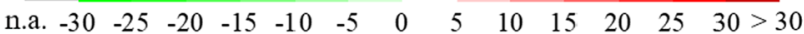

Fig. 2 Increases (red) and decreases (green) of municipal waste amount and composition in Montreal and Trento during the pandemic. The variations $\left(V_{i}^{j}, \%\right)$ of any type of waste $\left(W_{i}\right.$, tons) between a COVID-19 pandemic period (j) in 2020-2021 versus the same period in 2019 were calculated using Eq. 3: $V_{i}^{j}(\%)=\frac{{ }_{{ }_{C O V I D}{ }_{i}-W_{2019_{i}^{j}}}}{W_{2019_{i}^{j}}} \times 100 \%$. The red grids mean that the waste

commercial and construction wastes than household waste due to the drastic reduction in business, construction and tourist activities.

There were about $12 \%$ increases in the total waste amount in both March and June for Montreal, while nearly 9\% increases were all observed in February, June and September for Trento. Each type of Montreal's waste increased by about 11\% in March and June. However, Trento's organic waste increased by $10.6 \%$ in February and its packaging waste increased by $12.1 \%$ and $16.4 \%$ in June and September, respectively (Fig. 2). Besides, February-April 2020 and November 2020-January 2021 experienced the first lockdown and second lockdown periods, respectively. Thus, waste variations in Trento showed periodic changes under re-lockdown measures as that observed since the first lockdown period (Figs. 1 and 2). In the first month of lockdowns, the panic shopping of necessary items, inappropriate cooking habits, such as overcooking, broken food supply chains, packaged take-out orders and home-delivered businesses, might result in increased food and packaging wastes (Aldaco amount during the lockdown periods in 2020 increased compared to the normal periods in 2019 , whereas the green grids mean the decreases in waste amount. The gray grids mean no data available. The black vertical dashed lines show different lockdown periods in the pandemic

et al. 2020; Jribi et al. 2020). In addition, unlocking might cause many inhabitants to return to normal food habits and produce more food waste than the average (WRAP 2020). Thus, revenge consumption might explain June's increase in Montreal's MSW. Besides, the transition from in-store shopping to online shopping would increase the amount of in-home recyclable packaging waste (Ikiz et al. 2021). The visitors to retail \& recreation places and grocery \& pharmacy stores were still much fewer than those during the normal periods, even though the lockdown intensity was reduced to level 3 (Fig. 1; COVID-19 Community Mobility Reports 2021). This means that lockdown measures influenced consumers' behaviors during the pandemic, leading to an increase in online shopping and packaging wastes in Trento. These increases were observed in the first month of lockdowns and the second month of unlocking, likely because unlocking requires more response time. Overall, the initial implementation of lockdown or unlocking measures promoted inhabitants' consumption, generally leading to an increase in waste amount. Moreover, consumers' behaviors 
in different lockdown periods affect variations in waste amount and composition.

Besides people's movements and consumers' behaviors, waste management affects waste amount and composition. For example, MSW was generally collected more on Mondays, Tuesdays and Thursdays than on Wednesdays and Fridays in Montreal. Therefore, the significant monthly variations in Montreal's MSW amount were likely due to more "major collection weekdays" in March and June while less in April, May and July in 2020 compared to 2019. Considering the spreading risks of contaminated wastes in recycling services, some Euro-American areas, including the six selected countries, restricted selective collections, e.g., a 35.5\% reduction between March 23 and April 24 in Brazil, and recycling services, e.g., a $46 \%$ decrease in USA. Alternately, single-use plastic packaging was resurged, and recovery facilities were partially stopped in these countries. Consequently, the amount of recyclable waste generally decreased, but mixed and bulky wastes increased during March-April 2020 because of significant changes in waste management services during the early pandemic (Fan et al. 2020; Gorrasi et al. 2020; Ragazzi et al. 2020). Overall, larger variations in the waste amount in Trento were in line with higher lockdown intensity compared to those in Montreal, affected by people mobility, consumers' behaviors and waste management.

\section{Conclusion}

Our results showed the long-term effect of varying pandemic lockdowns on waste variations during different lockdown periods in some Euro-American regions during 2020-2021. Overall, the government lockdown intensity and people's movements changed with the varying lockdowns, resulting in variations in the amount and composition of MSW during the pandemic. Generally, there have been mainly four successive lockdown periods in 2020 and people mobility appears better controlled in Italy and France versus USA, Brazil, Canada, UK. We found prolonged lockdowns caused larger decreases in commercial and construction wastes than household waste due to the drastic reduction in business, construction and tourist activities. The beginning of lockdown or unlocking measures promoted inhabitants' consumption, generally leading to an increase in waste amount. Moreover, larger variations in the waste amount in Trento were in line with higher lockdown intensity compared to those in Montreal, affected by people mobility, consumers' behaviors and waste management. Notably, the inadequacies of waste management systems in the early pandemic may lead to a decrease in waste collection of recyclable waste but an increase of bulky and mixed wastes. Thus, an appropriate strategy for the whole waste management system, including waste generation, collection, transport, recycling and disposal, should be considered to reduce waste for incineration and direct landfilling and increase waste recycling. Furthermore, the use of some new approached such as the automated waste management system through the Internet of Things can alleviate the risk of labor shortage and workers' infection. Lastly, the resurgence of single-use plastic packaging is a temporary alternative, although it may cause further microplastic pollution and affect public engagement in MSW management after the pandemic. The recovery of waste management practices and policies based on a circular economy can be a priority.

Supplementary Information The online version contains supplementary material available at https://doi.org/10.1007/s10311-021-01290-z.

Authors' contribution Mengfan Cai conducted the study and wrote the article. Christophe Guy, Martin Héroux, Eric Lichtfouse, and Chunjiang An conceived the study and revised the article.

Funding This research was supported by the Natural Sciences and Engineering Research Council of Canada.

\section{Declarations}

Conflict of interest This is to certify that the authors of this manuscript have no affiliations with or involvement in any organization or entity with any financial interest, or non-financial interest in the subject matter or materials discussed in this manuscript.

\section{References}

Aldaco R, Hoehn D, Laso J et al (2020) Food waste management during the COVID-19 outbreak: a holistic climate, economic and nutritional approach. Sci Total Environ 742:140524. https://doi. org/10.1016/j.scitotenv.2020.140524

City of Montreal (2020) 2020 report on the management of residual materials in the agglomeration of Montreal. Available via. http://ville.montreal.qc.ca/pls/portal/docs/PAGE/ENVIRO_FR/ (unpublished)

COVID-19 Community Mobility Reports (2021) Community Mobility Reports. Available via. https://www.google.com/covid19/mobil ity/. Accessed 21 March 2021

Das AK, Islam MN, Billah MM et al (2021) COVID-19 and municipal solid waste (MSW) management: a review. Environ Sci Pollut Res. https://doi.org/10.1007/s11356-021-13914-6

Fan YV, Jiang P, Hemzal M et al (2020) An update of COVID-19 influence on waste management. Sci Total Environ 754:142014. https://doi.org/10.1016/j.scitotenv.2020.142014

Google News (2021) Coronavirus (COVID-19) statistics data. Available via. https://news.google.com/covid19/map?hl=en-CA\&gl= CA\&ceid=CA\%3Aen. Accessed 21 Mar 2021

Gorrasi G, Sorrentino A, Lichtfouse E (2020) Back to plastic pollution in COVID times. Environ Chem Lett 19:1-4. https://doi.org/10. 1007/s10311-020-01129-z

Huo C, Dar AA, Nawaz A et al (2021) Groundwater contamination with the threat of COVID-19: insights into CSR theory of Carroll's pyramid. J King Saud Univ Sci 33(2):101295. https://doi. org/10.1016/j.jksus.2020.101295 
Ikiz E, Maclaren VW, Alfred E et al (2021) Impact of COVID-19 on household waste flows, diversion and reuse: the case of multiresidential buildings in Toronto Canada. Resour Conserv Recycl. https://doi.org/10.1016/j.resconrec.2020.105111

Jribi S, Ben Ismail H, Doggui D et al (2020) COVID-19 virus outbreak lockdown: what impacts on household food wastage? Environ Dev Sustain 22(5):3939-3955. https://doi.org/10.1007/ s10668-020-00740-y

Kulkarni BN, Anantharama V (2020) Repercussions of COVID-19 pandemic on municipal solid waste management: challenges and opportunities. Sci Total Environ 743:140693. https://doi.org/10. 1016/j.scitotenv.2020.140693

OxCGRT (2021) Oxford Covid-19 Government Response Tracker. Available via. https://github.com/OxCGRT. Accessed 23 March 2021

Ragazzi M, Rada EC, Schiavon M (2020) Municipal solid waste management during the SARS-COV-2 outbreak and lockdown ease: Lessons from Italy. Sci Total Environ. https://doi.org/10.1016/j. scitotenv.2020.141159

Somani M, Srivastava AN, Gummadivalli SK et al (2020) Indirect implications of COVID-19 towards sustainable environment: an investigation in Indian context. Bioresour Technol Rep 11:100491. https://doi.org/10.1016/j.biteb.2020.100491

The Conversation (2020) COVID-19 is laying waste to many US recycling programs. Available via. https://theconversation.com/ covid-19-is-laying-waste-to-many-us-recycling-programs-139733. Accessed 23 June 2020

Tolvik (2020) Residual Waste during Q2 2020 lockdown. Available via. https://www.tolvik.com/residual-waste-during-q2-2020-lockd own/. Accessed 15 September 2020

Trento Municipality (2021) Data on the percentages of separate waste collection in recent years. Available via. https://www.comune. trento.it/Aree-tematiche/Ambiente-e-territorio/Rifiuti-urbani/ Gestione-integrata-rifiuti/Raccolta-differenziata/Risultati-raggi unti. Accessed January 2021

Vaverková MD, Paleologos EK, Dominijanni A et al (2020) Municipal solid waste management under COVID-19: challenges and recommendations. Environ Geotech 8(3):217-232. https://doi.org/10. 1680/jenge.20.00082

Vu HL, Ng KTW, Richter A et al (2021) Modeling of municipal waste disposal rates during COVID-19 using separated waste fraction models. Sci Total Environ 789:148024. https://doi.org/10.1016/j. scitotenv.2021.148024
Wang L, Li M, Yu S et al (2020a) Unexpected rise of ozone in urban and rural areas, and sulfur dioxide in rural areas during the coronavirus city lockdown in Hangzhou, China: implications for air quality. Environ Chem Lett 18(5):1713-1723. https://doi.org/10. 1007/s10311-020-01028-3

Wang X, Wang P, Wang C et al (2020b) Taxonomic and functional responses of sediment bacterial community to anthropogenic disturbances in the yarlung tsangpo river on the Tibetan Plateau. J Environ Inform 35(1):23-33. https://doi.org/10.3808/jei.20180 0403

Wang Z, An C, Chen X et al (2021) Disposable masks release microplastics to the aqueous environment with exacerbation by natural weathering. J Hazard Mater 417:126036. https://doi.org/10.1016/j. jhazmat.2021.126036

Waste Advantage (2020) The impact of COVID-19 on the waste and recycling industry. Available via. https://wasteadvantagemag. com/the-impact-of-covid-19-on-the-waste-and-recycling-indus try/. Accessed 1 May 2020

WRAP (2020) Food waste and Covid-19 - Survey 2: Lockdown easing. Available via. https://wrap.org.uk/resources/report/citizens-andfood-waste-lockdown-eases. Accessed July 2020

You S, Sonne C, Ok YS (2020) COVID-19's unsustainable waste management. Science 368(6498):1438

Yousefi M, Oskoei V, Jafari AJ et al (2021) Municipal solid waste management during COVID-19 pandemic: effects and repercussions. Environ Sci Pollut Res. https://doi.org/10.1007/ s11356-021-14214-9

Zambrano-Monserrate MA, Ruano MA, Sanchez-Alcalde L (2020) Indirect effects of COVID-19 on the environment. Sci Total Environ 728:138813. https://doi.org/10.1016/j.scitotenv.2020.138813

Zand AD, Heir AV (2020) Emerging challenges in urban waste management in Tehran, Iran during the COVID-19 pandemic. Resour Conserv Recycl 162:105051. https://doi.org/10.1016/j.resconrec. 2020.105051

Publisher's Note Springer Nature remains neutral with regard to jurisdictional claims in published maps and institutional affiliations. 